ISSN: $1679-3013$

D.O.I.: 10.5914/to.2014.01186

\title{
ICTIOFAUNA DAS PISCINAS DE MARÉ DA PRAIA DO PAIVA: UM REGISTRO ANTERIOR À EXPLORAÇÃO IMOBILIÁRIA
}

Recebido em: $29 / 09 / 2014$

Aceito em: 22/10/2014

\author{
Rafael dos Santos DANTAS ${ }^{1}$ \\ Patrícia Silva Basílio DANTAS 2,3 \\ Robson Tamar da Costa RAMOS ${ }^{1}$
}

\begin{abstract}
RESUMO
Piscinas de maré são importantes berçários para peixes recifais e estão constantemente sujeitas a impactos, principalmente oriundos de ação humana. O objetivo deste trabalho foi gerar um banco de dados acerca da ictiofauna da Praia do Paiva, uma área de piscinas de maré bem conservadas e sob ameaça antrópica. Foram amostradas três piscinas abertas e duas fechadas através de

de 66 espécies foi observado nas piscinas estudadas, muitas delas com importância socioeconômica. Embora a composição taxonômica registrada seja semelhante àquela de outros estudos na costa Nordeste, o número de espécies foi alto, quando comparado a outras áreas de piscinas desta região, o que evidencia a importância da Praia do Paiva como amostra conservada da biodiversidade.
\end{abstract} transecto de faixa e busca ativa. Um total

Palavras-chave: Conservação, Desenvolvimento Urbano, Peixes Recifais.

\section{ABSTRACT}

Tide pools are important nurseries for reef fish and are constantly subject to impacts, mainly arising from human action. The objective of this work was to generate a database about the Paiva Beach ichthyofauna, a well preserve tide pool area under anthropogenic threat. Three open and two closed pools were sampled through transect and active searching. A total of 66 fish species was observed in the studied pools, many with socioeconomic importance. Although the registered taxonomic composition was similar to other studies in the Northeastern coast the number of species was high when compared to other areas with similar characteristics, which highlights the importance of Paiva Beach as a wellpreserved sample of the biodiversity.

Keywords: Conservation, Urban Development, Reef fishes.

\section{INTRODUÇÃO}

As piscinas de maré são hábitats típicos de costas rochosas e são caracterizados por elevada complexidade estrutural (ZANDER; NIEDER; MARTIN, 1999). Nesses ambientes, a água permanece em cavidades e depressões que funcionam como refúgio para muitos peixes cuja fisiologia suporta as variações físico-químicas que caracterizam as marés (DANTAS, 2013). Além disso, as piscinas também são reconhecidas como berçários de várias espécies de peixes recifais (BENNETT; GRIFFITHS, 1984; BECKLEY, 1985; BENNETT, 1987).

Estes ambientes estão sujeitos a diversos impactos, como a pesca destrutiva (WATSON et al., 1996), exploração para fins de aquariofilia (NOTTINGHAM et al., 2005), efeito do desenvolvimento costeiro (OLIVEIRA et al., 2009), atividades turísticas não planejadas (ILARRI et al., 2007), entre outros. Tais ameaças são ainda mais graves quando consideramos que as piscinas são corpos d'água de pequeno porte, o que amplifica e concentra os impactos.

A Praia do Paiva fica inserida em uma região que está sob influência direta do complexo portuário de Suape, e, no período no qual foram realizadas as coletas de dados deste trabalho, ainda podia ser considerada um ambiente conservado e pouco habitado (DANTAS, 2013). A

\footnotetext{
${ }^{1}$ PPGCB - CAMPUS I, DSE, Universidade Federal da Paraíba, João Pessoa, PB

${ }^{2}$ ITEP - Associação Instituto de Tecnologia de Pernambuco, Recife, PE

${ }^{3}$ PPGEMA - CAMPUS IV, Universidade Federal da Paraíba, Rio Tinto, PB
} 
DANTAS, R.S.; DANTAS, P.S.B.; RAMOS, R.T.C. Ictiofauna das piscinas de maré da praia do Paiva: um registro anterior à exploração imobiliária

praia está localizada em um extenso braço de terra conhecido como "Ilha do Amor" e possuía como forma de acesso principal a travessia realizada por canoas e pequenas embarcações (SACRAMENTO; ZICKEL; ALMEIDA JR., 2007). No ano de 2010 foi inaugurada uma ponte de acesso às praias do litoral sul pernambucano (Gaibu, Calhetas, Porto de Galinhas, etc.), e o processo de urbanização ali iniciado incluiu a construção de vias urbanas, conjuntos habitacionais e complexos hoteleiros.

Nos últimos anos, a construção civil cresceu em ritmo acelerado e novos projetos para a construção de resorts e condomínios de luxo estão sendo executados, um deles nas margens da faixa de praia onde se encontram as piscinas estudadas (Rafael Dantas, observação pessoal). Sendo assim, a urgência na execução de levantamentos das comunidades se eleva, tendo em vista que a crescente exploração da região pode trazer desequilíbrio ambiental e suprimir espécies. A realização deste trabalho tem como motivação a geração de um banco de dados acerca da ictiofauna local, com o objetivo de preservar testemunho do ambiente ainda em estado conservado, antes que a exploração imobiliária possa gerar as consequências que acompanha comumente este tipo de fenômeno antropológico.

\section{ÁREA DE ESTUDO}

A região litorânea do Estado de Pernambuco encontra-se na faixa de clima tropical úmido que se estende do litoral Potiguar ao litoral Baiano. As temperaturas dessa região variam entre 23 e $32^{\circ} \mathrm{C}$, com precipitação média de $1500 \mathrm{~mm} /$ ano (APAC, 2014). Duas estações são bem definidas: uma seca (Primavera e Verão), de Setembro a Março, e outra chuvosa (Outono e Inverno), de Abril a Julho.

A região metropolitana do Recife (RMR) possui uma linha de costa com aproximadamente $60 \mathrm{~km}$, que engloba as praias do Paiva, Candeias, Piedade, Boa Viagem e Pina, entre outras, com temperatura da água variando entre 24 e $30^{\circ} \mathrm{C}$, durante o verão (FISCHER et al., 2009). A praia do Paiva (Erro! Fonte de referência não encontrada.) abrange uma área de aproximadamente 553 ha (SACRAMENTO; ZICKEL; ALMEIDA JR., 2007) e está localizada no Cabo de Santo Agostinho, um dos municípios que fazem parte da RMR. Está inserida em uma região sob influência direta do estuário formado pelos rios Jaboatão e Pirapama (NORIEGA et al., 2005).

As piscinas estudadas nesse trabalho estão localizadas na zona mediolitoral da costa pernambucana, dentro do complexo recifal da praia do Paiva, classificadas como piscinas abertas (nomeadas A1, A2 e A3) e fechadas (nomeadas F1 e F2). Foram consideradas piscinas abertas aquelas que não perdem conexão com o mar, mesmo em marés muito baixas. Por possuírem tal característica, essas piscinas tendem a apresentar uma menor variação da temperatura e mudanças menos drásticas nas condições físico-químicas da água. Espera-se também que ocorra um fluxo de indivíduos entrando e saindo da piscina, principalmente de carnívoros e herbívoros não territorialistas, em busca de alimento. Deve-se notar, entretanto, que a capacidade de locomoção que as espécies possuem não é mesma, e espera-se, portanto, que esse fluxo não funcione igualmente em todas elas. Foram classificadas como fechadas as piscinas que ficam totalmente cercadas por formações rochosas que ocasionam uma desconexão hídrica de superfície com o mar aberto, durante o período de baixa-mar. São elas que apresentam os efeitos característicos do isolamento de corpos d'água no ambiente recifal, tais como aumento da temperatura e salinidade e outras mudanças físico-químicas acentuadas da água (DAVENPORT; WOOLMINGTON, 1981). 


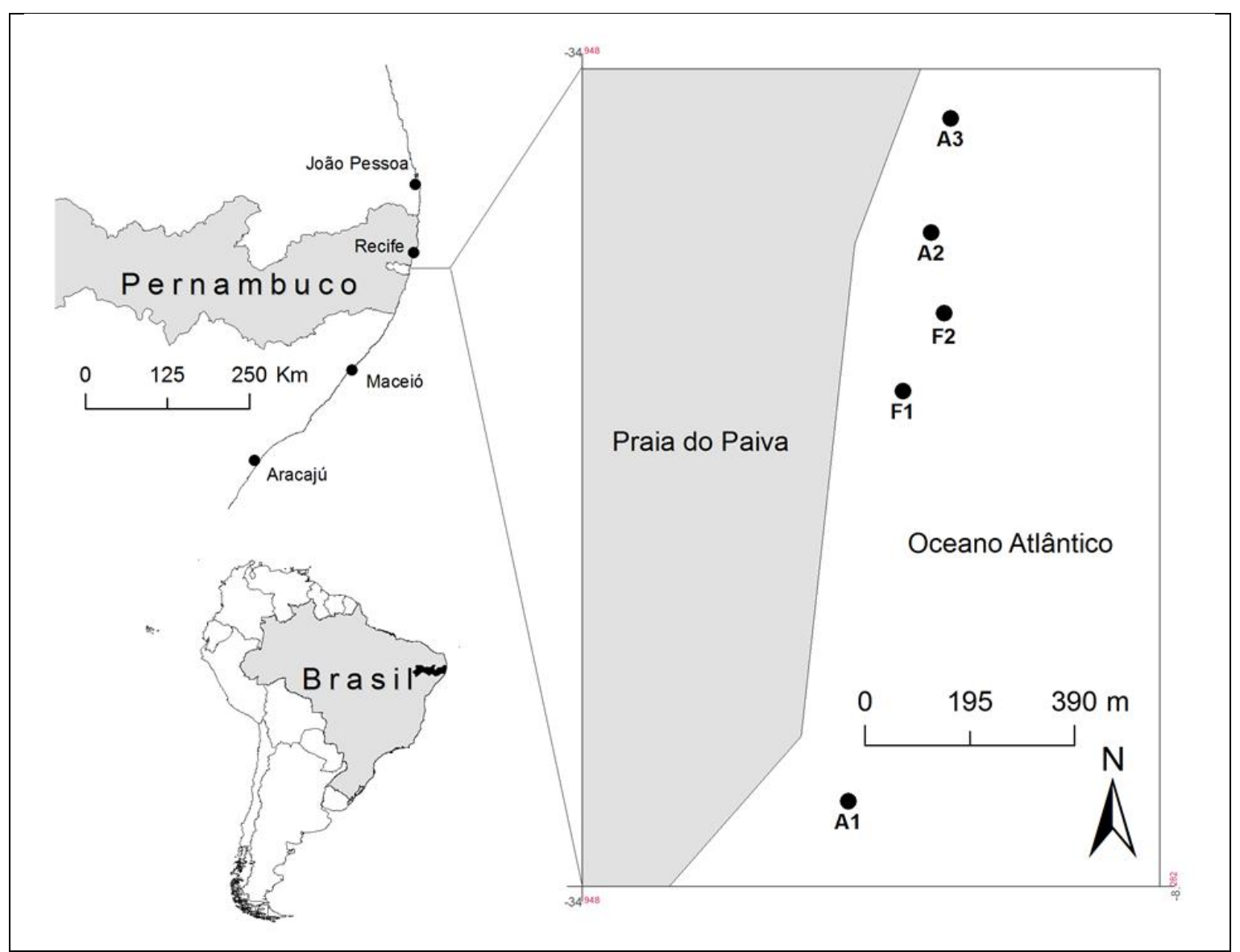

Figura 1 - Área de estudo e seu posicionamento geográfico; no detalhe, a praia do Paiva, Cabo de Santo Agostinho, PE, com destaque para localização das piscinas estudadas. (A1, A2 e A3: piscinas abertas 1, 2 e 3; F1 e F2, piscinas fechadas 1 e 2)

\section{Posição geográfica e caracterização morfológica das Piscinas}

Piscina Aberta 1 (A1, Erro! Fonte de referência não encontrada.A): localizada a $08^{\circ} 16^{\prime} 49.0^{\prime \prime}$ S $34^{\circ} 56^{\prime} 45.8^{\prime \prime} \mathrm{W}$, é uma formação com área aproximada de $240 \mathrm{~m}^{2}$, conectada ao oceano pela face leste, profundidade média de 0,9 metro; Piscina Aberta 2 (A2, Erro! Fonte de referência não encontrada.B): localizada a $08^{\circ} 16^{\prime} 14.6^{\prime \prime} \mathrm{S} 34^{\circ} 56^{\prime} 40.8^{\prime \prime} \mathrm{W}$, compreende área aproximada de $1080 \mathrm{~m}^{2}$, permanentemente conectada com o mar, mesmo nas marés mais baixas, profundidade média de 0,7 metro; Piscina Aberta 3 (A3, Erro! Fonte de referência não encontrada.C): localizada a $08^{\circ} 16^{\prime} 07.7^{\prime \prime} \mathrm{S} 34^{\circ} 56^{\prime} 39.6^{\prime \prime} \mathrm{W}$, área aproximada em $440 \mathrm{~m}^{2}$, profundidade média observada de 0,75 metro, conexão com o oceano se dá na face nordeste, através de uma passagem rasa (aproximadamente 0,2 metro) e estreita (10 metros de largura); Piscina Fechada 1 (F1, Erro! Fonte de referência não encontrada.D): localizada a $08^{\circ} 16^{\prime}$ $24.2^{\prime \prime} \mathrm{S} 34^{\circ} 56^{\prime} 42.5^{\prime \prime} \mathrm{W}$, corresponde a uma área aproximada de $320 \mathrm{~m}^{2}$, profundidade média de 0,85 metro: Piscina Fechada 2 (F2, Erro! Fonte de referência não encontrada.E): localizada a $08^{\circ} 16^{\prime} 19.5^{\prime \prime} \mathrm{S}, 34^{\circ} 56^{\prime} 40.0^{\prime \prime} \mathrm{W}$, área de aproximadamente $960 \mathrm{~m}^{2}$, profundidade média de 0,98 metro. 


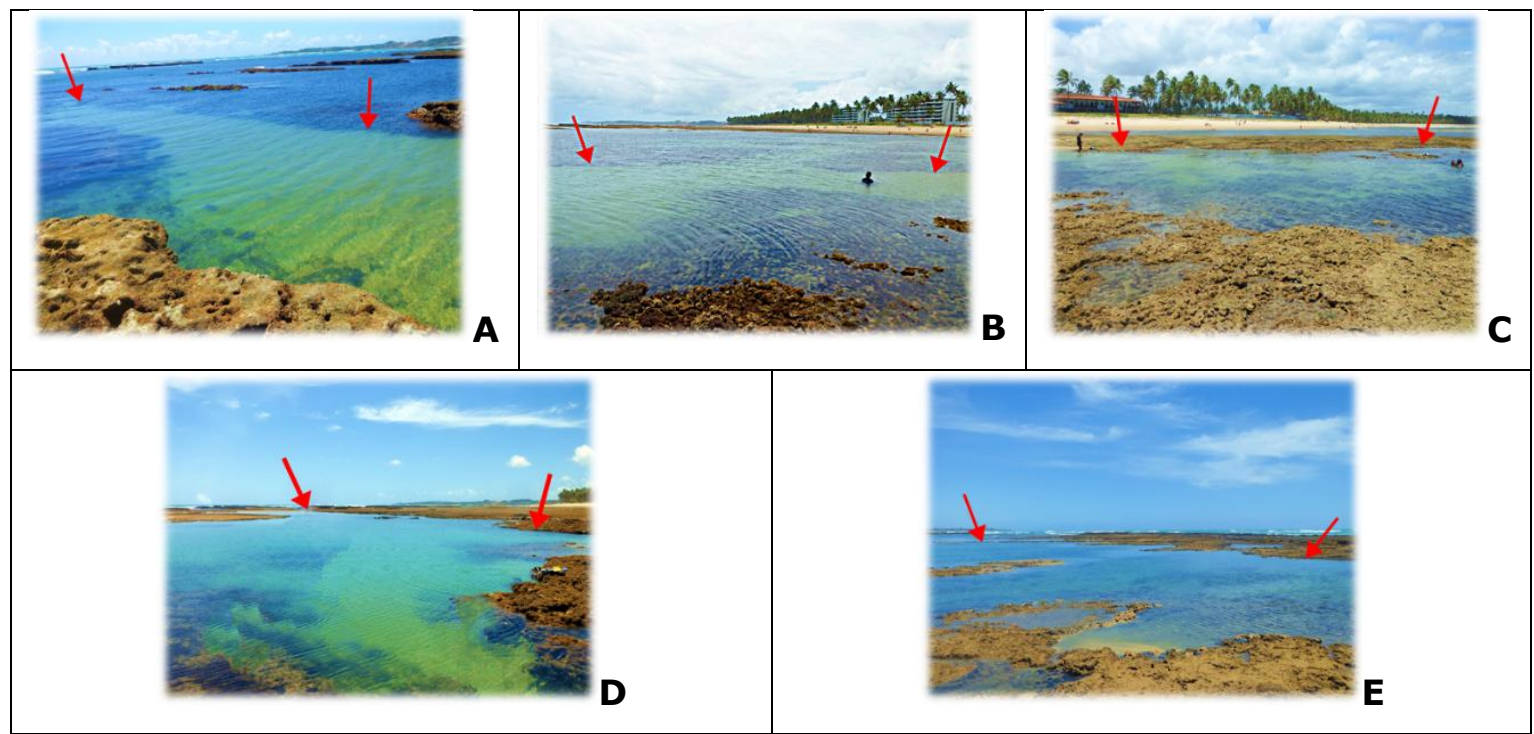

Figura 2 - Visão das Piscinas estudadas. A: A1; B: A2; C: A3; D: F1; E: F2. As setas vermelhas indicam os limites das piscinas. Fotos: Dantas, R.S.

\section{MATERIAL E MÉTODOS}

A coleta de dados foi realizada em expedições diurnas, durante marés baixas de sizígia, no período compreendido entre os meses de janeiro e junho de 2012.

A amplitude das marés foi obtida consultando a tábua disponível no endereço eletrônico www.mar.mil.br/dhn/chm/tabuas/index.htm, usando como base o complexo portuário de Suape. Como os mergulhos foram realizados na baixa-mar, o início das atividades aconteceu sempre 30 minutos antes do horário do estofo de baixa-mar, estimado pela Diretoria de Hidrografia e Navegação, a partir do website destacado acima.

Foi utilizada a metodologia adaptada do Reefcheck, com transecto de faixa para peixes. Este método consiste na utilização de trenas de $10 \mathrm{~m}$ de comprimento ao longo da qual são contados (Censo Visual) os indivíduos que estiverem 2 metros à direita e 2 metros à esquerda da trena (Erro! Fonte de referência não encontrada.). Ao longo da trena, o mergulhador anotou em uma prancha de PVC as espécies observadas. As amostragens foram iniciadas 5 minutos após a colocação da trena, com a finalidade de reduzir a influência da presença do pesquisador sobre o objeto amostrado.

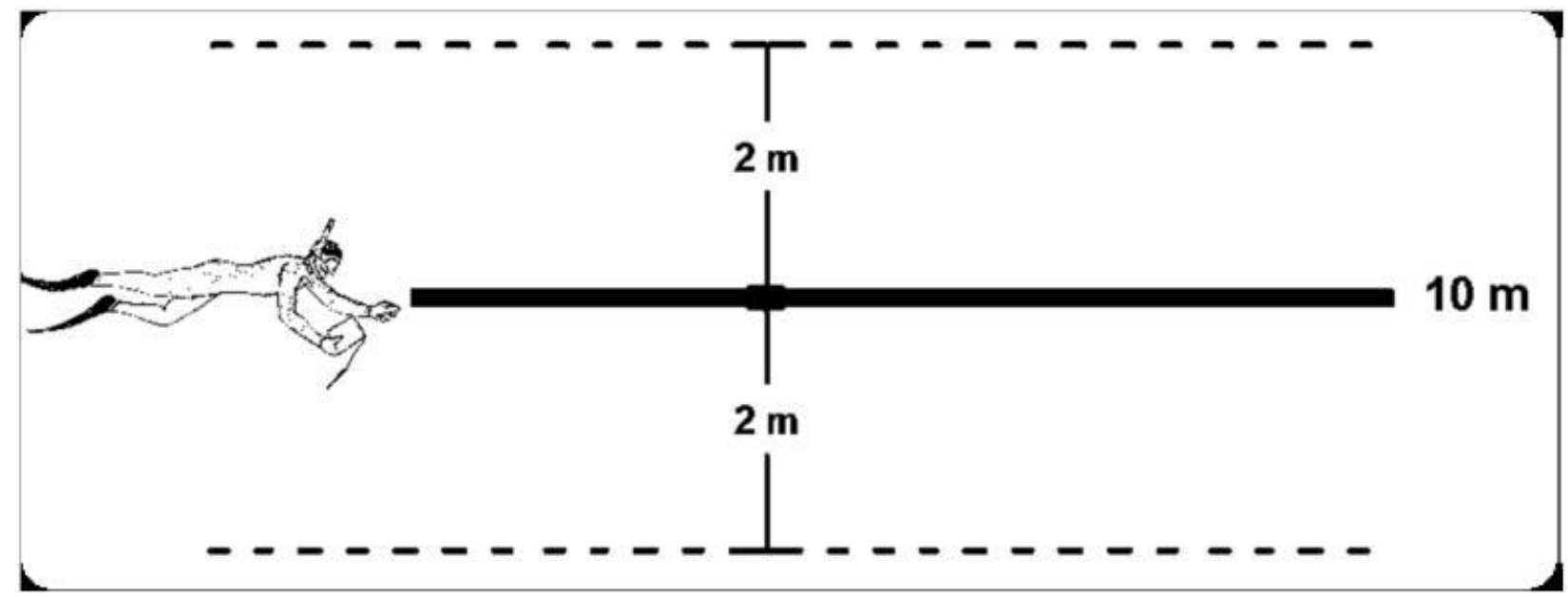

Figura 3 - Desenho esquemático da metodologia de censo visual através de transectos realizados nas piscinas de maré da praia do Paiva, Cabo de Santo Agostinho, PE. Ilustração: Helder C. Lima 
Foi calculada a curva de acumulação de espécies, com objetivo de confrontar os dados obtidos através da metodologia do censo visual com alguns estimadores de diversidade (Bootstrap e Chao1), essa etapa foi conduzida utilizando o software EstimateS.

O objetivo deste trabalho foi coletar o máximo possível de informações acerca de quais espécies de peixes recifais ocorrem na área a ser estudada. Para tanto, foram também realizadas observações livres (busca ativa) em todos os locais amostrados para complementar qualitativamente o registro de espécies não observadas através do método de transecto/censo visual. A técnica de busca ativa consiste no rastreamento de toda a área, explorando a maior diversidade de habitats possíveis, anotando-se as espécies avistadas. Também as coletas de busca ativa foram conduzidas sempre pelo mesmo mergulhador e se iniciaram com pelo menos 30 minutos de antecedência ao horário de baixa-mar, de forma a permitir ao pesquisador ter tempo suficiente para a realização das observações. Foi feita uma exploração exaustiva de todas as regiões acessíveis das piscinas, registrando as espécies encontradas nas pranchas de PVC

Foi realizada a identificação visual em campo, ao seu nível específico, seguindo literatura especializada (HUMANN; DELOACH, 2002; REEFNET, 2007). Os indivíduos dos quais não foi possível a identificação visual em campo foram fotografados através de câmera digital acoplada a caixa estanque para posterior identificação no laboratório.

\section{RESULTADOS}

Durante o período de coletas foram realizados 75 censos visuais em transectos distribuídos nas cinco piscinas selecionadas, perfazendo $3000 \mathrm{~m}^{2}$ de área amostrada. A curva de acumulação de espécies realizada pelo Estimates mostra que houve um rápido aumento do número de espécies registradas entre os transectos 1 e 25 , e que a curva tendeu à estabilidade a partir deste ponto da amostragem. Foi possível observar também que, após o transecto 50, $91,37 \%$ das espécies observadas já tinham sido registradas. O estimador Bootstrap indicou a existência de 48,17 espécies (valor real: 46), ao passo que o estimador Chao1 indicou 46,61 espécies. Da mesma forma, a partir do transecto 50, estes estimadores evidenciaram 90,37\% e $93,22 \%$ das espécies, respectivamente (Figura 4)

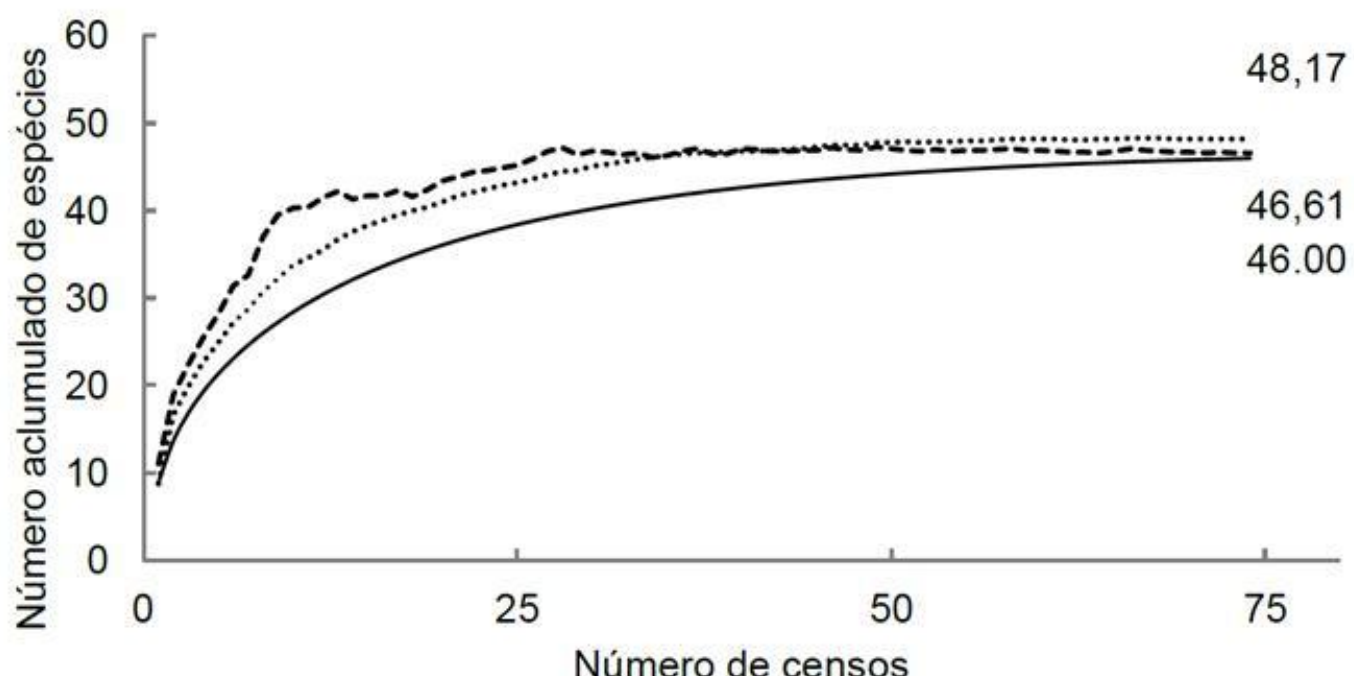

- Riqueza observada $\quad$-----Chao $1 \quad$.......... Bootstrap

Figura 4 - Número acumulado de espécies de peixes na praia do Paiva, Cabo de Santo Agostinho, $\mathrm{PE}$, em função do número total de censos realizados nas piscinas estudadas e projeções dos estimadores Chao1 e Bootstrap. 
Considerando as amostragens somadas de transectos e busca ativa, foram registradas 66 espécies pertencentes a 45 gêneros, 34 Famílias e 13 ordens (Erro! Fonte de referência não encontrada.).

Tabela 1 - Lista das espécies registradas nas piscinas de maré da praia do Paiva, Cabo de Santo Agostinho, PE. Status IUCN: DD; Dados insuficientes; NT: Quase Ameaçada; LC: Pouco Preocupante; EM: Em Perigo; Vazias: Não consta na lista vermelha. A ordem das espécies segue classificação proposta por Nelson (2006)

\begin{tabular}{|c|c|c|}
\hline Família/Espécie & $\begin{array}{l}\text { Status } \\
\text { IUCN }\end{array}$ & $\begin{array}{c}\text { Uso } \\
\text { Socioeconômico }\end{array}$ \\
\hline \multicolumn{3}{|l|}{ Dasyatidae } \\
\hline Dasyatis americana Hildebrand \& Schroeder, 1928 & DD & $x$ \\
\hline \multicolumn{3}{|l|}{ Albulidae } \\
\hline Albula vulpes (Linnaeus, 1758) & NT & $x$ \\
\hline \multicolumn{3}{|l|}{ Muraenidae } \\
\hline Gymnothorax funebris Ranzani, 1839 & & $x$ \\
\hline Gymnothorax moringa (Cuvier, 1829) & & $x$ \\
\hline Gymnothorax vicinus (Castelnau, 1855) & & $x$ \\
\hline \multicolumn{3}{|l|}{ Ophichthidae } \\
\hline \multicolumn{3}{|l|}{ Myrichthys ocellatus (Lesueur, 1825) } \\
\hline \multicolumn{3}{|l|}{ Synodontidae } \\
\hline \multicolumn{3}{|l|}{ Synodus sp. } \\
\hline \multicolumn{3}{|l|}{ Batrachoididae } \\
\hline \multicolumn{3}{|l|}{ Thalassophryne punctata Steindachner, 1876} \\
\hline \multicolumn{3}{|l|}{ Mugilidae } \\
\hline Mugil curema Valenciennes, 1836 & & $x$ \\
\hline Mugil liza Valenciennes, 1836 & & $x$ \\
\hline \multicolumn{3}{|l|}{ Atherinopsidae } \\
\hline Atherinella brasiliensis (Quoy \& Gaimard, 1825) & & $x$ \\
\hline \multicolumn{3}{|l|}{ Hemiramphidae } \\
\hline Hemiramphus sp. & & $x$ \\
\hline \multicolumn{3}{|l|}{ Holocentridae } \\
\hline Holocentrus adscensionis (Osbeck, 1765) & & $x$ \\
\hline Myripristis jacobus Cuvier, 1829 & & $x$ \\
\hline \multicolumn{3}{|l|}{ Scorpaenidae } \\
\hline \multicolumn{3}{|l|}{ Scorpaena brasiliensis Cuvier, 1829} \\
\hline Scorpaena plumieri Bloch, 1789 & LC & \\
\hline \multicolumn{3}{|l|}{ Serranidae } \\
\hline Cephalopholis fulva (Linnaeus, 1758) & LC & $x$ \\
\hline Epinephelus adscensionis (Osbeck, 1765) & LC & $x$ \\
\hline Mycteroperca bonaci (Poey, 1860) & NT & $x$ \\
\hline \multicolumn{3}{|l|}{ Apogonidae } \\
\hline \multicolumn{3}{|l|}{ Apogon americanus Castelnau, 1855} \\
\hline \multicolumn{3}{|l|}{ Carangidae } \\
\hline Carangoides bartholomaei (Cuvier, 1833) & & $\mathrm{x}$ \\
\hline Caranx latus Agassiz, 1831 & & $\mathrm{x}$ \\
\hline Oligoplites saurus (Bloch \& Schneide, 1801) & & $\mathrm{x}$ \\
\hline \multicolumn{3}{|l|}{ Lutjanidae } \\
\hline Lutjanus alexandrei Moura \& Lindeman, 2007 & & $x$ \\
\hline Lutjanus jocu (Bloch \& Schneider, 1801) & & $x$ \\
\hline \multicolumn{3}{|l|}{ Gerreidae } \\
\hline \multicolumn{3}{|l|}{ Eucinostomus melanopterus (Bleeker, 1863) } \\
\hline \multicolumn{3}{|l|}{ Haemulidae } \\
\hline Anisotremus moricandi (Ranzani, 1842) & EN & $x$ \\
\hline
\end{tabular}


DANTAS, R.S.; DANTAS, P.S.B.; RAMOS, R.T.C. Ictiofauna das piscinas de maré da praia do Paiva: um registro anterior à exploração imobiliária

Anisotremus surinamensis (Bloch, 1791)

Anisotremus virginicus (Linnaeus, 1758)

Haemulon aurolineatum Cuvier, 1830

Haemulon parra (Desmarest, 1823)

Haemulon plumieri (Lacepède, 1801)

Haemulon squamipinna Rocha \& Rosa, 1999

Haemulon steindachneri (Jordan \& Gilbert, 1882)

Sciaenidae

Odontoscion dentex (Cuvier, 1830)

Pareques acuminatus (Bloch \& Schneider, 1801)

\section{Mullidae}

Mulloidichthys martinicus (Cuvier, 1859)

Pseudupeneus maculatus (Bloch, 1793)

\begin{tabular}{cc} 
& $\mathrm{x}$ \\
$\mathrm{DD}$ & $\mathrm{x}$ \\
& $\mathrm{x}$ \\
& $\mathrm{x}$ \\
& $\mathrm{x}$ \\
\hline LC & $\mathrm{x}$
\end{tabular}

Pempheridae

Pempheris schomburgkii Müller \& Troschel, 1848

\section{Chaetodontidae}

Chaetodon striatus Linnaeus, 1758

LC

Pomacanthidae

Pomacanthus paru (Bloch, 1787)

LC

$x$

Pomacentridae

Abudefduf saxatilis (Linnaeus, 1758)

Microspathodon chrysurus (Cuvier, 1830)

Stegastes fuscus (Cuvier, 1830)

Stegastes variabilis (Castelnau, 1855)

\section{Labridae}

Halichoeres brasiliensis (Bloch, 1791)

Halichoeres poeyi (Steindachner, 1867)

$\mathrm{x}$

Scaridae

Sparisoma axillare (Steindachner, 1878)

Sparisoma frondosum (Agassiz, 1831)

Sparisoma radians (Valenciennes, 1840)

$\mathrm{x}$

$x$

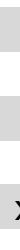

Blenniidae

Ophioblennius trinitatis Ribeiro, 1919

Labrisomidae

Labrisomus nuchipinnis (Quoy \& Gaimard, 1824)

Malacoctenus sp.

Malacoctenus triangulatus Springer, 1959

Gobiidae

Coryphopterus glaucofraenum Gill, 1863

Ephippidae

Chaetodipterus faber (Broussonet, 1782)

Acanthuridae

Acanthurus bahianus Castelnau, 1855

Acanthurus chirurgus (Bloch, 1787)

Acanthurus coeruleus Bloch \& Schneider, 1801

LC

Sphyraenidae

Sphyraena barracuda (Edwards, 1771)

Bothidae

Bothus lunatus (Linnaeus, 1758)

DD

LC

Tetraodontidae

Sphoeroides greeleyi Scopoli, 1777

$\begin{array}{ll}\text { DD } & x \\ \text { DD } & x \\ \text { LC } & x\end{array}$

LC

$x$

Sphoeroides spengleri (Bloch, 1785)

Sphoeroides testudineus (Linnaeus, 1758)

$x$

Diodontidae

Tropical Oceanography, Recife, v. 42, n. 2, p. 253-260, 2014. 
DANTAS, R.S.; DANTAS, P.S.B.; RAMOS, R.T.C. Ictiofauna das piscinas de maré da praia do Paiva: um registro anterior à exploração imobiliária

Diodon holocanthus Linnaeus, 1758

Diodon hystrix Linnaeus, 1758

\section{DISCUSSÃO}

Bohnsack e Bannerot (1986) afirmam que são necessários, no mínimo, vinte amostras para descrever algumas comunidades com censos visuais. A Figura 4 mostra que a estabilização da curva acumulada de espécies se deu a partir de 25 amostras, corroborando a afirmação desses autores. Além disso, os estimadores de diversidade reforçam a percepção de que a amostragem nesse estudo foi adequada para descrever a comunidade íctica local.

De modo geral, a composição taxonômica registrada nesse trabalho foi semelhante àquela encontrada em outros estudos realizados na costa Nordeste do Brasil (FERREIRA; MAIDA; SOUZA, 1995; ROSA; ROSA; ROCHA, 1997; FEITOSA; PIMENTA; ARAÚJO, 2002; CUNHA et al., 2008; CHAVES; NUNES; SAMPAIO, 2010; GODINHO; LOTUFO, 2010; HONÓRIO; RAMOS; FEITOZA, 2010), uma constatação esperada, dada a localização e a dimensão da área estudada. O número de espécies encontradas (66), levando-se em consideração a busca ativa e a amostragem por transectos, foi relativamente alto, quando comparado a outras áreas de piscinas de maré da região nordeste, as quais variam entre 29 e 56 espécies (ROSA; ROSA; ROCHA, 1997; FEITOSA; PIMENTA; ARAÚJO, 2002; CUNHA et al., 2008; CHAVES; NUNES; SAMPAIO, 2010; GODINHO; LOTUFO, 2010). .

Horn et al. (1999) afirmam que a maior parte dos peixes de recifes costeiros utiliza, uma vez ou outra, ambientes na zona entre marés com diferentes propósitos, como alimentação e proteção. Estas espécies são classificadas como residentes de uma determinada área quando se utilizam desta por todo o seu ciclo de vida, sendo classificadas como residente secundárias quando as utiliza em apenas parte do ciclo. A prevalência neste estudo de famílias comumente encontradas em ambientes do infralitoral (Haemulidae, Scaridae e Acanthuridae, por exemplo) e a presença maciça de indivíduos juvenis destas (obs. pessoal), pode indicar que a maior parte das espécies registradas nas piscinas da praia do Paiva são residentes secundárias. Assim, é possível supor que estas espécies utilizam as piscinas estudadas como berçários. A importância de piscinas de maré como berçários já foi amplamente estudada em diversos locais do mundo, e isso só vem a reforçar o valor que a área de estudo tem para o ambiente costeiro da RMR.

Mesmo com essa destacada importância, o uso, sem qualquer controle, das piscinas por turistas e pescadores artesanais é evento constante observado na área de estudo. Cunha et al. (2008) listam vários impactos causados por humanos que podem afetar a diversidade de peixes de piscinas de maré, entre elas o turismo e a construção civil próxima da linha da costa, este último como um dos mais prejudiciais. O número de turistas que visitam a Praia do Paiva cresce exponencialmente, saltando de 4 mil no verão de 2012 para mais de 8 mil no verão de 2014 ("Novas regras ambientais na Praia do Paiva", 2014), o que pode gerar uma série de impactos à fauna local (SARMENTO; BARRETO; SANTOS, 2011). Além disso, em todas as expedições foi notada a presença de pescadores locais utilizando diversas artes de pesca. Na piscina F2, por exemplo, foi observada pesca de arrasto em uma das expedições. Somando-se a isso a crescente exploração imobiliária da praia do Paiva, é possível afirmar que a área está em perigo de perda de diversidade a curto e médio prazo.

O governo local, em parceria com o grupo empresarial responsável pela implementação e manutenção dos empreendimentos imobiliários na região, lançou recentemente um programa de ordenamento do uso da praia, com o objetivo de tentar mitigar os impactos gerados pelo aumento do uso do ambiente ("Novas regras ambientais na Praia do Paiva", 2014). Esse ordenamento prevê a proibição da permanência sobre os recifes (evitando, assim, o pisoteio), restrições em relação à pesca, entre outras medidas mais comuns, como a proibição de jogar lixo na faixa de areia. Entretanto, impactos já estabelecidos provavelmente não serão revertidos com essa ação, e o risco da perda de diversidade não foi afastado

\section{CONCLUSÕES}


- A ictiofauna recifal das piscinas da praia do Paiva é composta por espécies com distribuição pan-atlântica e tropical. A maioria das espécies está restrita ao Atlântico Ocidental, padrão comumente observado em estudos realizados ao longo da costa Nordeste do Brasil.

- Foi possível verificar que a diversidade registrada no ambiente estudado foi superior àquela observada em outros ambientes da costa brasileira, entre estes alguns protegidos por lei, sugerindo que a área estudada é, de fato, uma amostra importante dos ambientes de piscinas de maré brasileiros. Este estudo poderá servir de subsídio para futuro monitoramento da área em questão, pois se trata do único registro formal da diversidade e composição da ictiofauna recifal na praia do Paiva, hoje em franco processo de exploração imobiliária.

\section{REFERÊNCIAS BIBLIOGRÁFICAS}

APAC. Agência Pernambucana de Águas e Climas. Agência Governamental. Disponível em: <http://www.apac.pe.gov.br/>. Acesso em: 22 ago. 2014.

BECKLEY, L. E. The Fish Community of East Cape Tidal Pools And an Assessment of The Nursery Function of This Habitat. South African Journal of Zoology, v. 20, p. 21-27, 1985.

BENNETT, B. A. The rock-pool fish community of Koopie Alleen and an assessment of the importance of Cape rock-pools as nurseries for juvenile fish. South African Journal of Zoology, v. 22, p. 25-32, 1987.

BENNETT, B. A.; GRIFFITHS, C. L. Factors affecting the distribution, abundance and diversity of rock-pool fishes on the Cape peninsula, South-Africa. South African Journal of Zoology, v. 19, 1984.

BOHNSACK, J. A.; BANNEROT, S. P. A stationary visual census technique for quantitatively assessing community structure of coral reef fishes. United States: United States National Marine Fisheries Service, 1986.

CHAVES, L. DE C. T.; NUNES, J. DE A. C. C.; SAMPAIO, C. L. S. Shallow reef fish communities of South Bahia coast, Brazil. Brazilian Journal of Oceanography, v. 58, p. 33-46, 2010.

CUNHA, E. A. et al. Comparative analysis of tidepool fish species composition on tropical coastal rocky reefs at State of Ceará, Brazil. Iheringia. Série Zoologia, v. 98, p. 379-390, 2008.

DANTAS, R. S. Composição e Estrutura Trófica da Ictiofauna de Piscinas de Maré da praia do Paiva, Região Metropolitana do Recife, PE. Dissertação-João Pessoa/PB: DSE Universidade Federal da Paraíba, 2013.

DAVENPORT, J.; WOOLMINGTON, A. D. Behavioural responses of some rocky shore fish exposed to adverse environmental conditions. Marine Behaviour and Physiology, v. 8, p. 1$12,1981$.

FEITOSA, C. V.; PIMENTA, D. A. S.; ARAÚJO, M. E. Ictiofauna Recifal dos Parrachos de Maracajaú (RN) Na Área dos Flutuantes: Inventário e Estrutura da Comunidade. Arquivos das Ciências do Mar, v. 35, p. 39-50, 2002.

FERREIRA, B. P.; MAIDA, M.; SOUZA, A. E. T. Levantamento inicial das comunidades de peixes recifais da região de Tamandaré - PE. Boletim Técnico do CEPENE, v. 3, p. 213-230, 1995.

FISCHER, A. F. et al. Biological aspects of sharks caught off the Coast of Pernambuco, Northeast Brazil. Brazilian Journal of Biology, v. 69, p. 1173-1181, 2009.

GODINHO, W. O.; LOTUFO, T. M. C. Local v. microhabitat influences on the fish fauna of tidal pools in north-east Brazil. Journal of Fish Biology, v. 76, p. 487-501, 2010.

HONÓRIO, P. P. F.; RAMOS, R. T. C.; FEITOZA, B. M. Composition and structure of reef fish communities in Paraíba State, north-eastern Brazil. Journal of Fish Biology, v. 77, p. 907926, 2010.

HORN; KAREN L. M MARTIN; MICHAEL A. CHOTKOWSKI. Intertidal Fishes: Life in Two Worlds. 1. ed. San Diego: Academic Press, 1999. 
DANTAS, R.S.; DANTAS, P.S.B.; RAMOS, R.T.C. Ictiofauna das piscinas de maré da praia do Paiva: um registro anterior à exploração imobiliária

HUMANN, P.; DELOACH, N. Reef fish identification Florida-Caribbean-Bahamas. 3. ed. Florida: New World Publications, 2002.

ILARRI, M. et al. Recife de Picãozinho: um aquário natural ameaçado. Ciência Hoje, v. 41, n. 243, p. 70-72, 2007.

NELSON, J. S. Fishes of the World. 4th ed. ed. Hoboken, New Jersey: John Wiley \& Sons, Inc., 2006.

NORIEGA, C. D. et al. Fluxos de nutrientes inorgânicos dissolvidos em um estuário tropical Barra das Jangadas - PE, Brasil. Tropical Oceanography, v. 33, p. 133-145, 2005.

NOTTINGHAM, M. C. et al. Ordenamento da explotação de peixes ornamentais marinhos no Brasil. Boletim Técnico do CEPENE, v. 13, p. 75-107, 2005.

Novas regras ambientais na Praia do Paiva. Diário de Pernambuco. Disponível em: <http://www.diariodepernambuco.com.br/app/noticia/vidaurbana/2014/07/23/interna_vidaurbana,517882/novas-regras-ambientais-na-praia-dopaiva.shtml>. Acesso em: 22 set. 2014.

OLIVEIRA, A. et al. Percepção dos Ambientes Recifais da Praia de Boa Viagem (Recife/PE) por estudantes, Professores e Moradores. OLAM - Ciência \& Tecnologia, v. 9, n. 2, p. 136-163, 2009.

REEFNET, I. Reef fish ID: Florida, Caribbean, Bahamas. Florida, USA: [s.n.].

ROSA, R. S.; ROSA, I. L.; ROCHA, L. A. Diversidade da ictiofauna de poças de maré da praia do Cabo Branco, João Pessoa, Paraíba, Brasil. Revista Brasileira de Zoologia, v. 14, p. 201-212, 1997.

SACRAMENTO, A. C.; ZICKEL, C. S.; ALMEIDA JR., E. B. Aspectos florísticos da vegetação de restinga no litoral de Pernambuco. Revista Árvore, v. 31, p. 1121-1130, 2007.

SARMENTO, V. C.; BARRETO, A. F. S.; SANTOS, P. J. P. The response of meiofauna to human trampling on coral reefs. Scientia Marina, v. 75, n. 3, p. 559-570, 26 abr. 2011.

WATSON, M. et al. The Effects of Fishing on Coral Reef Fish Abundance and Diversity. Journal of the Marine Biological Association of the United Kingdom, v. 76, p. 229-233, 1996.

ZANDER, C. D.; NIEDER, J.; MARTIN, K. L. M. Vertical Distribution Patterns. In: HORN, M. H.; MARTIN, K. L. M.; CHOTKOWSKI, M. A. (Eds.). . Intertidal Fishes: Life in Two Worlds. 1. ed. San Diego: Academic Press, 1999. p. 26-54. 